K.A. van der Hucht, G. Koenigsberger \& P.R.J. Eenens, eds.

\title{
WR 146 - observing the OB-type companion
}

\author{
Sean M. Dougherty \\ University of Calgary/Dominion Radio Astrophysical Observatory NRC, \\ P.O. Box 248, Penticton, B.C. V2A 6K3, Canada
}

Peredur M. Williams

Royal Observatory, Blackford Hill, Edinburgh, Scotland EH9 зHJ, UK

\begin{abstract}
Using both MERLIN and the VLA, we have succeeded in detecting the radio emission from three components in WR 146: the WR star, the wind-wind collision region, and for the first time, the stellar companion to the WR star. This allows the unique possibility of determining the mass loss rate ratio of the two winds, an important observable that is independent of distance.
\end{abstract}

\section{Introduction}

Previous MERLIN observations at $5 \mathrm{GHz}$ of WR 146 show two components, $\mathrm{N}_{5}$ and $S_{5}$, separated by $\sim 120$ mas (Dougherty et al. 1996). $S_{5}$ is thermal and is identified as the free-free emitting envelope around the WR star, whereas $N_{5}$ is non-thermal. An optical spectrum reveals an OB-type companion, leading to the suggestion that the non-thermal emission arises from relativistic electrons in the region where the wind of the WR star and the OB-type companion interact (e.g., Eichler \& Usov 1993). Using the HST, Niemela et al. (1998) identified a visual companion to the WR star 170 mas from WR 146, somewhat further from WR 146 than the non-thermal emission region, confirming the wind-wind collision as the origin of the non-thermal emission.

\section{New observations}

New observations were obtained with the high resolution A-configuration of the $V L A$ at $22 \mathrm{GHz}$. These observations reveal two components, $\mathrm{N}_{22}$ and $\mathrm{S}_{22}$, very similar in appearance to the MERLIN $5-\mathrm{GHz}$ data. The flux of $\mathrm{S}_{22}$ is $7.0 \pm 1.3 \mathrm{mJy}$, consistent with that expected from the free-free emitting stellar wind from the WR star with a spectral index of $+0.74 \pm 0.2$. We conclude that $S_{22}$ and $S_{5}$ are both the stellar wind of the WR star. The separation of the $22-\mathrm{GHz}$ components is $162 \pm 8$ at a position angle of $22^{\circ}$, consistent with the relative separation $(168 \pm 31 \mathrm{mas})$ and position angle $\left(21 \pm 4^{\circ}\right)$ of optical components WR 146A and B observed by the HST (Niemela et al. 1998). From a simple proper motion study of $\mathrm{N}_{5}$ and $\mathrm{S}_{5}$ observed by MERLIN at two earlier epochs we conclude that $\mathrm{N}_{5}$ and $\mathrm{N}_{22}$ are not the same feature. This leads us to suggest that the two components detected in the $22-\mathrm{GHz}$ observations are the two stellar components imaged by the HST.

The relative position of the three components. gives the momentum ratio of the two winds, $\eta$, and the flux ratio of the emission from both stellar winds gives 


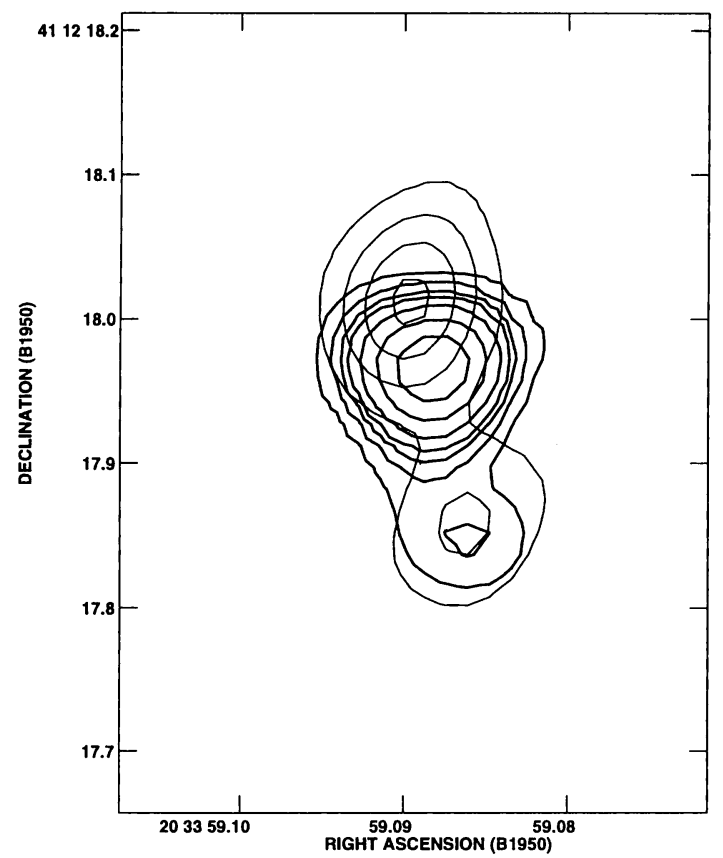

Figure 1. Overlay of the VLA 22-GHz image (thin contours) with a MERLIN 5-GHz image obtained in 1992 (thick contours), assuming $\mathrm{S}_{22}$ and $\mathrm{S}_{5}$ are coincident. Clearly, the northern component at $22 \mathrm{GHz}$ is displaced to the north of the non-thermal region observed at $5 \mathrm{GHz}$.

the density ratio of the two winds, $\xi$. Note that both $\eta$ and $\xi$ are independent of distance and inclination, though $\xi$ is dependent on the wind composition, particularly the mean atomic weight $\mu$. Taking reasonable values of $\mu$, we find that $\eta=0.11 \pm 0.03$ and $\xi=0.47 \pm 0.25$. From these values, the ratio of mass loss rates of the two stars $\sim 0.25$, a value independent of distance. This is about an order of magnitude larger than expected for an $\mathrm{O}$ star wind relative to that of a WR star. For the derived ratio to be smaller, $\xi$ has to decrease, which implies that we have to attribute some of the flux of $\mathrm{N}_{22}$ to the wind collision region. For example, if we assume that the terminal velocities of the winds are the same $(\xi=\eta)$, then the wind flux from the $\mathrm{O}$ star companion would be $\sim 2$ $3 \mathrm{mJy}$, implying that 7-8 mJy of the flux observed in $\mathrm{N}_{22}$ comes from the wind collision zone. If this is the case, the coincidence of the peak of the $22-\mathrm{GHz}$ emission with the location of the companion, as observed by the HST, presents a different puzzle.

\section{References}

Dougherty, S.M., Williams, P.M., van der Hucht, K.A., Bode, M.F., Davis, R.J. 1996, MNRAS 280, 963

Eichler, D., Usov, V.V. 1993, ApJ 402, 271

Neimela, V.S., Shara, M.M., Wallace, D.J., Zurek, D.R., Moffat, A.F.J. 1998, AJ 115, 2047 